ARTICLE

DOI: $10.1038 / \mathrm{s} 41467-018-02955-0$

\title{
Selenide-catalyzed enantioselective synthesis of trifluoromethylthiolated tetrahydronaphthalenes by merging desymmetrization and trifluoromethylthiolation
}

\author{
Jie Luo ${ }^{1}$, Qingxiang Cao ${ }^{1}$, Xiaohui Cao $^{1} \&$ Xiaodan Zhao (1) ${ }^{1}$
}

Trifluoromethylthiolated molecules are an important class of biologically active compounds and potential drug candidates. Because of the lack of efficient synthetic methods, catalytic enantioselective construction of these molecules is rare and remains a challenge. To expand this field, we herein disclose a bifunctional selenide-catalyzed approach for the synthesis of various chiral trifluoromethylthiolated tetrahydronaphthalenes bearing an all-carbon quaternary stereocenter with gem-diaryl-tethered alkenes and alkynes by merging desymmetrization and trifluoromethylthiolation strategy. The products are obtained in high yields with excellent enantio- and diastereo-selectivities. This method can be applied to the desymmetrization and sulfenylation of diols as well. Computational studies reveal that selenide can activate the electrophilic reagent better than sulfide, confirming the higher efficiency of selenide catalysis in these reactions. On the basis of the theoretical calculations, an acidderived anion-binding interaction is suggested to exist in the whole pathway and accounts for the observed high selectivities.

\footnotetext{
${ }^{1}$ Institute of Organic Chemistry and MOE Key Laboratory of Bioinorganic and Synthetic Chemistry, School of Chemistry, Sun Yat-Sen University, Guangzhou 510275, PR China. Correspondence and requests for materials should be addressed to X.C. (email: caoxh5@mail.sysu.edu.cn)

or to X.Z. (email: zhaoxd3@mail.sysu.edu.cn)
} 
$\mathrm{n}$ recent years, many efforts have been devoted to the incorporation of fluorine atoms or fluorine-containing groups such as trifluoromethyl $\left(\mathrm{CF}_{3}\right)$, trifluoromethoxy $\left(\mathrm{CF}_{3} \mathrm{O}\right)$, and trifluoromethanesulfenyl $\left(\mathrm{CF}_{3} \mathrm{~S}\right)$ ones into the parent molecules for various purposes because of the fluorine effect ${ }^{1-6}$. Among these endeavors, strategic synthesis of $\mathrm{CF}_{3} \mathrm{~S}$ molecules has been paid special attention owing to the strong electron-withdrawing effect and extremely high lipophilicity value $\left(\pi_{\mathrm{R}}=1.44\right)$ of $\mathrm{CF}_{3} \mathrm{~S}$ group ${ }^{5-}$ 12. However, little success has been achieved on enantioselective trifluoromethylthiolation until now, although stereogenic $\mathrm{CF}_{3} \mathrm{~S}$ molecules warrant further studies considering the importance of chiral centers in medicine ${ }^{13-20}$. Thus, developing new methods to create versatile chiral $\mathrm{CF}_{3} \mathrm{~S}$ molecules, especially those with an allcarbon quaternary stereocenter through a novel and enantioselective reaction mode, is highly desirable.

Catalytic enantioselective desymmetrization is an attractive strategy for the construction of chiral all-carbon quaternary stereocenters by the conversion of prochiral quaternary carbon centers $^{21-23}$. Using this strategy, numerous valuable, potentially bioactive molecules having a chiral all-carbon quaternary center can be quickly accessed from different functionlized starting materials ${ }^{24-43}$. In particular, olefinic or alkynyl carboxylic acids $^{33,34}$, alcohols ${ }^{35-39}$, and amines ${ }^{40-43}$ were frequently employed as the substrates to undergo enantioselective desymmetrization and cyclization to generate heterocycles by metal- or organocatalysis (Fig. 1a). In these transformations, the tethered nucleophile played an important role that it could bind a catalyst to guarantee an effective attack toward the multiple bond, which led to the formation of chiral products with high enantioselectivities. In contrast, enantioselective desymmetrization involving the attack of aryl group toward a multiple bond that results in the formation of multisubstituted tetrahydronaphthalene derivatives, an important class of bioactive compounds ${ }^{44-46}$, has been far less explored possibly because of the lack of the appropriate interaction between the aryl moiety and catalyst ${ }^{47-49}$. Only a few relevant examples have been reported by Chemler who utilized amine- or hydroxy-tethered alkenes for carboamination and etherification through a copper-catalyzed radical pathway (Fig. 1b) ${ }^{50-53}$.

Continuing our interest in Lewis basic selenium ${ }^{54-62}$-catalyzed trifluoromethylthiolation ${ }^{19,20,63-65}$, we intended to produce chiral $\mathrm{CF}_{3} \mathrm{~S}$ molecules with an all-carbon quaternary stereocenter through an enantioselective, electrophilic desymmetrization, and trifluoromethylthiolation mode. We envisioned that when gemdiaryl-tethered alkenes were employed as the substrates, the aryl group on substrate could act as a nucleophile to attack chiral selenide-captured trifluoromethylthiiranium moiety to directly afford chiral $\mathrm{CF}_{3} \mathrm{~S}$ tetrahydronaphthalenes (Fig. 1c). To cope with the main difficulty in this transformation, a proper chiral catalyst is essential that can control the attacking environment of the aryl group and thus induce the enantioselectivity of multiprochiral centers. Herein, we report our effort that gem-diaryl-tethered alkenes can undergo enantioselective desymmetrization and difunctionalization to efficiently afford $\mathrm{CF}_{3} \mathrm{~S}$ tetrahydronaphthalene derivatives with bifunctional selenide catalyst. The generated products contain one chiral quaternary carbon center and other two stereocenters. The developed method can be applied to enantioselective desymmetrization and sulfenylation of diols as well.

\section{Results}

Initial Attempts and Optimization of Reaction Conditions. We began our study of the electrophilic desymmetrization with 2,2diphenyl olefinic benzamide $\mathbf{1 a}$ as the model substrate. It could be easily synthesized from diphenylacetonitrile, and possesses two phenyl groups as a nucleophile and an extra benzamide group. To test the desymmetrization of 1a, highly reactive electrophilic $\left(\mathrm{PhSO}_{2}\right)_{2} \mathrm{NSCF}_{3}$ as the $\mathrm{CF}_{3} \mathrm{~S}$ source and bifunctional catalyst $\mathbf{C 1}$ based on indane scaffold were utilized (Table 1). Based on our former observations ${ }^{20}$, selenide $\mathbf{C 1}$ with a triflic amide group was quite efficient for the trifluoromethylthiolation with the aid of acid. Pleasingly, at room temperature, the corresponding product 2a was smoothly formed rather than amination product from benzamide group in $94 \%$ nuclear magnetic resonance (NMR) yield with $89 \%$ ee and 5:1 $d r$ using trimethylsilyl trifluoromethylsulfonate (TMSOTf) as the acid. Lowering the reaction temperature to $-78^{\circ} \mathrm{C}$ could quickly improve the enantioselectivity to $97 \%$ ee with unchanged diastereoselectivity (Table 1, entry 2). It is noted that sulfide catalyst C2 was not effective for this transformation at all under the similar conditions (Table 1, entry 3). To improve the diastereoselectivity of $2 \mathbf{a}$, various aryl selenides based on $\mathbf{C 1}$ were tested for the reaction. While para-substituted phenyl group and meta-substituted phenyl group on the selenide had little influence, ortho-substituent on the phenyl ring largely enhanced the selectivity (Table 1, entries 5-8). To our delight, catalyst C7 bearing both orthomethyl and methoxy groups was highly efficient to afford $2 \mathbf{a}$ in 99\% yield with 99\% ee and 50:1 $d r$. Using the mixed solvents of $\mathrm{CH}_{2} \mathrm{Cl}_{2}$ and $\left(\mathrm{CH}_{2} \mathrm{Cl}\right)_{2}$, the enantioselectivity of product $2 \mathrm{a}$ could be improved to $>99 \%$ (Table 1, entry 9). In addition, other acids including both Lewis acid or BrØnsted acid gave slightly lower enantioselectivity (Table 1, entries 10-12). It is noteworthy that the reaction could not go to completion and the corresponding product was formed in moderate selectivity under the optimal conditions when the substrate derived from $\mathbf{1 a}$ by further a

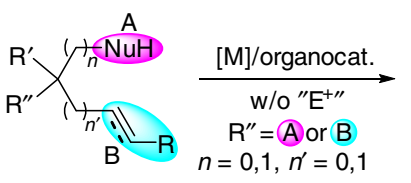

C

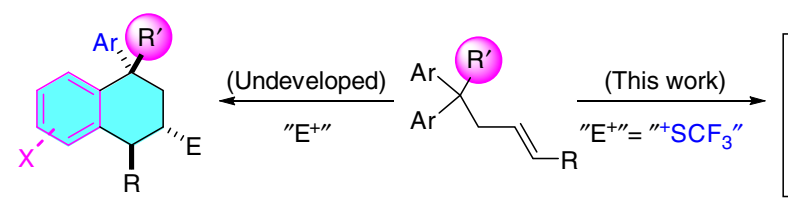

b

$\mathrm{Nu}-\mathrm{H}: \mathrm{Ar}$ Few examples:
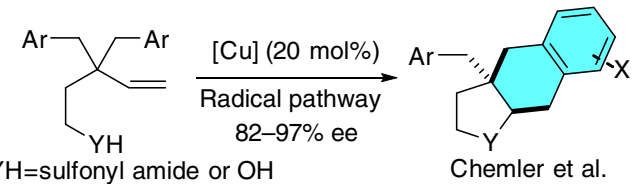

Chemler et al.
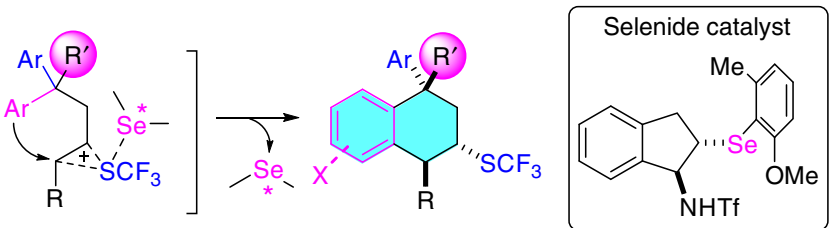

Fig. 1 Enantioselective construction of all-carbon quaternary center-containing molecules via desymmetrization. a Known strategies for enantioselective desymmetrization. b Desymmetrization through copper-catalyzed radical pathway. c Enantioselective desymmetrization and trifluoromethylthiolation using aryl group as a nucleophile 


\section{Table 1 Screening of reaction conditions}

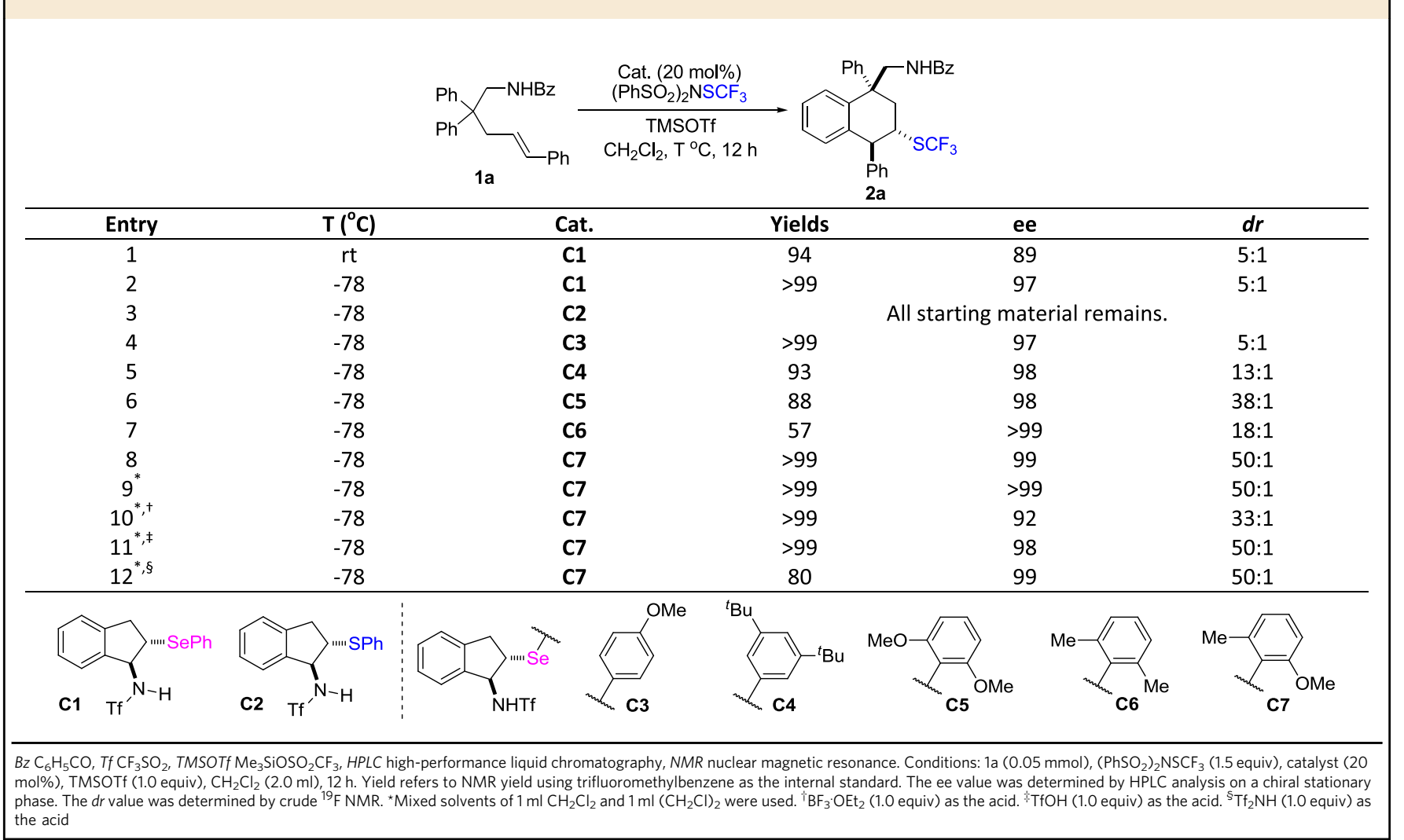

protecting nitrogen with methyl group was used (63\% ee, see Supplementary Table 3 for details).

Desymmetrization and Trifluoromethylthiolation. With the optimal conditions in hand, we began to explore the substrate scope (Table 2). To ensure the full consumption of starting materials, $20 \mathrm{~mol} \%$ of the catalyst loading was utilized for the transformations. Various aryl substituted olefins were first tested. All of them gave the corresponding products in good to excellent yields with excellent enantio- and diastereoselectivities (2a-h, 74-99\% yields, 98-99\% ees). Moreover, modified conditions were required for some substrates to give better yields or slightly better enantioselectivities. For example, the reactions could not go to completion under the optimal conditions for the formation of 2b-2d most likely because the weakly electron-withdrawing aryl group on the double bond eroded its reactivity toward $\mathrm{CF}_{3} \mathrm{~S}$ cation. When the reaction temperature was raised to $-60^{\circ} \mathrm{C}$, all these substrates were fully converted. Besides, low catalyst loading (10 mol\%) and low concentration were appropriate for the generation of $2 \mathbf{e}$ and $\mathbf{2 h}$ to suppress the possible attack of the electron-rich aryl group of catalyst toward the iranium ion. It was worthy to mention that a substrate bearing ortho-methyl-substituted phenyl group still gave the desired product in excellent yield with excellent enantioselectivity in spite of the steric hindrance around the double bond (2f, 94\% yield, $>99 \%$ ee). Enantioselective desymmetrizaiton of alkyl-substituted olefins was carried out under the similar conditions. Substrates bearing methyl or phenylethyl group gave the corresponding products in good yields with excellent ees (2i, $97 \%$ ee; $\mathbf{2 j}$, 97\% ee). To our surprise, gem-dialkyl-substituted olefins could efficiently afford the products bearing another achiral quaternary carbon center with excellent enantioselectivities (2k, 92\% ee; $\mathbf{2 1}$, 97\% ee), although large steric hindrance might affect the cyclization.
Moreover, the developed method was also suitable for alkynederived compounds. Olefinic products were obtained in good yields. When phenyl-substituted substrate was utilized in the reaction, product $\mathbf{2} \mathbf{m}$ was formed with excellent ee (95\% ee). The ethyl-substituted substrate gave 2 n with a little lower ee (87\%). These products contain a double bond, which can provide an opportunity for their further transformations. The absolute configuration of products was assigned to be $1 R, 3 S, 4 S$ based on the X-ray crystallographic study of $\mathbf{2 a}$.

The effect of functional groups attached to the quaternary carbon center on substrates was investigated (Table 2). When substrate 10 with more acidic proton was used, the reaction proceeded efficiently to afford the carbocyclization product 2o. In contrast, when the nitrogen of 10 was protected by methyl group, the corresponding substrate $\mathbf{1 0}^{\prime}$ gave product $\mathbf{2 0}^{\prime}$ with lower enantioselectivity $(85 \%$ ee $)$. It was noted that when the phenyl group attached to the double bond on 10 was replaced by an alkyl group, $\mathrm{CF}_{3} \mathrm{~S}$-amination product was observed along with the formation of carbocyclization product. Free hydroxyl group on substrate had an impact on the enantioselectivity (2p, $81 \%$ ee). Compared to the reaction of 4-nitro-benzenesulfonamide (NsNH)-functionalized substrate, the decrease of enantioselectivity might attribute to $\mathrm{OH}$-induced inappropriate $\mathrm{H}$-bonding interaction between substrate and catalyst. When the hydroxyl group was protected by benzoyl or acyl group, the cyclization proceeded efficiently to produce the products with excellent ees (2q-s, 94-97\% ees). It was noteworthy that the reaction of $1 \mathbf{q}$ was incomplete and afforded product $\mathbf{2 q}$ with $96 \%$ ee at $-78{ }^{\circ} \mathrm{C}$. Unexpectedly, when $\mathrm{R}^{\prime}$ group was hydrogen, the desired product 2t was still generated in $81 \%$ yield with $86 \%$ ee.

We then turned our attention to the desymmetrization with different gem-diaryl-tethered alkenes. Substrates with para- or ortho-substituted phenyl group at the quaternary carbon center gave the products in high yields with $>99 \%$ ees under the similar 


\section{Table 2 Enantioselective desymmetrization and trifluoromethylthiolation of $\mathbf{g e m}$-diaryl tethered alkenes/alkynes}

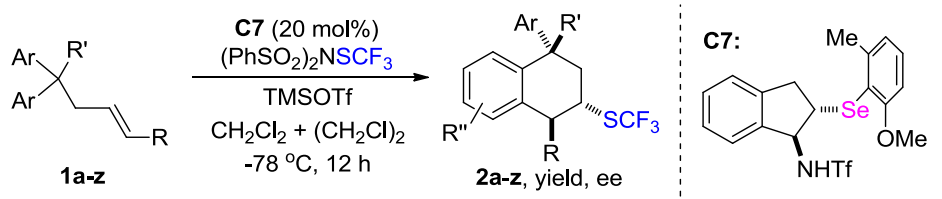

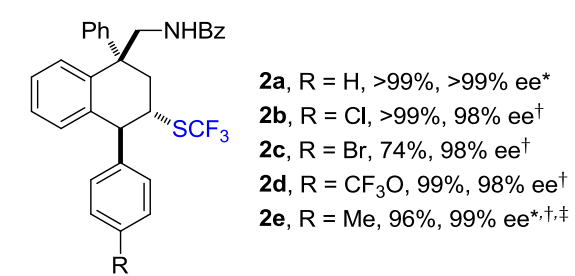
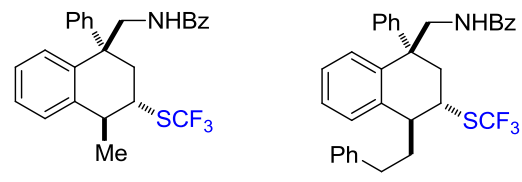

2i, $71 \%, 97 \%$ ee<smiles>O=[N+]([O-])CC1(c2ccccc2)C[C@H](C(F)(F)F)C(c2ccccc2)c2ccccc21</smiles>

2o, $\mathrm{X}=\mathrm{H}, 91 \%, 94 \% \mathrm{ee}^{\dagger, \text {, }}$ $20^{\prime}, X=M e, 75 \%, 85 \%$ ee<smiles>Cc1ccc([C@]2(CNC(=O)c3ccccc3)C[C@H](C(F)(F)F)C(c3ccccc3)c3cc(C)ccc32)cc1</smiles>

$2 u,>99 \%,>99 \%$ ee 2j, $80 \%, 97 \%$ ee*<smiles>OC[C@]1(c2ccccc2)C[C@H](C(F)(F)F)[C@H](P)c2ccccc21</smiles>

2p, $75 \%, 79 \%$ ee<smiles>O=C(NC[C@@]1(c2ccc(-c3ccccc3)cc2)C[C@H](C(F)(F)F)[C@H](c2ccccc2)c2cc(P)ccc21)C(F)(F)F</smiles>

$2 \mathbf{v}, 86 \%,>99 \% e^{*}$

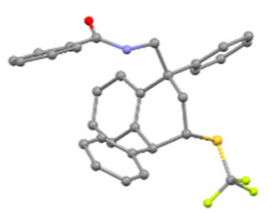

X-ray for $\mathbf{2 a}$<smiles>CC(=O)NC[C@@]1(c2ccccc2)C[C@H](C(F)(F)F)C(C)(C)c2ccccc21</smiles>

2k, $99 \%, 92 \%$ ee<smiles>CC(C)(C)OCC[C@@]1(c2ccccc2)C[C@H](C(F)(F)F)C(c2ccccc2)c2ccccc21</smiles>

2q, $79 \%, 94 \% \mathrm{ee}^{\star, \dagger}$<smiles>COc1cccc([C@]2(CNC(=O)c3ccccc3)C[C@H](C(F)(F)F)[C@H](c3ccccc3)c3ccc(OC)cc32)c1</smiles>

2w, $52 \%,>99 \%$ ee

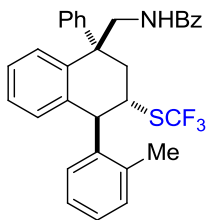

2f, $94 \%$, >99\% ee<smiles>O=C(NC[C@@]1(c2ccccc2)C[C@H](C(F)(F)F)C2(CCCC2)c2ccccc21)c1ccccc1</smiles>

2I, $68 \%, 97 \%$ ee<smiles>C[C@@H]1c2ccccc2[C@@](COC(C)(C)C)(c2ccccc2)C[C@@H]1C(F)(F)F</smiles>

2 r, $95 \%, 97 \%$ ee<smiles>Cc1cccc([C@]2(CNCC(=O)c3ccccc3)C[C@H](C(F)(F)F)[C@H](c3ccccc3)c3ccc(C)cc32)c1</smiles>

$2 x, 85 \%,>99 \%$ ee<smiles>COc1cccc(C2c3ccccc3C(CNC(=O)c3ccccc3)(c3ccccc3)C[C@H]2C(F)(F)F)c1</smiles>

2g, 96\%, 99\% ee<smiles>O=C(NC[C@@]1(c2ccccc2)CC([Si](F)(F)F)=C(c2ccccc2)c2ccccc21)C(F)(F)F</smiles>

$2 \mathrm{~m}, 68 \%, 95 \% \mathrm{ee}^{\S}$<smiles>CC(=O)OC[C@@]1(c2ccccc2)C[C@H](C(F)(F)F)C(C)c2ccccc21</smiles>

2s, $78 \%, 97 \%$ ee<smiles>Cc1cccc2c1[C@@](CNC(=O)c1ccccc1)(c1ccccc1)C[C@H](C(F)(F)F)[C@H]2c1ccccc1</smiles>

$2 y, 71 \%,>99 \% \mathrm{ee}^{\dagger}$

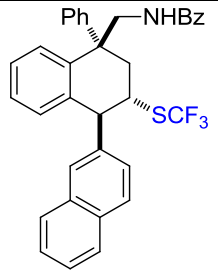

2h, 97\%, 99\% ee ee,,$\ddagger^{*}$<smiles>CCC1=C([Si](F)(F)F)C[C@@](CNC(=O)c2ccccc2)(c2ccccc2)c2ccccc21</smiles>

2n, $73 \%, 87 \% \mathrm{ee}^{\S}$<smiles>FC(F)(F)[C@H]1C[C@H](c2ccccc2)c2ccccc2[C@H]1c1ccccc1</smiles>

2t, $84 \%, 83 \%$ ee<smiles>CC1c2cccc3c2[C@](CCOC(C)(C)C)(C[C@@H]1C(F)(F)F)c1ccccc1-3</smiles>

2z $89 \%, 90 \%$ ee

93\%, 94\% ee

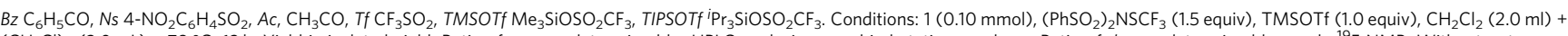
$\left(\mathrm{CH}_{2} \mathrm{Cl}\right)_{2}(2.0 \mathrm{~mL}),-78^{\circ} \mathrm{C}, 12 \mathrm{~h}$. Yield is isolated yield. Ratio of ee was determined by HPLC analysis on a chiral stationary phase. Ratio of $d r$ was determined by crude ${ }^{19} \mathrm{~F} \mathrm{NMR}$. Without note, diastereoselectivity is >99:1. *With $50: 1$ diastereoselectivity. ${ }^{\dagger}$ Reaction temperature: $-60^{\circ} \mathrm{C}^{*}{ }^{+} \mathrm{CH}_{2} \mathrm{Cl}_{2}(4.0 \mathrm{ml})+\left(\mathrm{CH} \mathrm{Cl}_{2}(4.0 \mathrm{ml})\right.$ as the solvent; $10 \mathrm{~mol} \%$ catalyst was used. ${ }^{\S} \mathrm{TMSOTf}(2.0$ equiv) was added. /With 8:1 diastereoselectivity. "TIPSOTf (1.0 equiv) instead of TMSOTf. " $\mathrm{BF}_{3} \cdot \mathrm{OEt}_{2}$ (2.0 equiv) instead of TMSOTf

conditions (2u, 2v, and $\mathbf{2 y})$. When substrates with metasubstituted phenyl group at the quaternary carbon center were utilized, regioisomeric products were formed because of the site selectivity. The major isomer could be isolated with extremely high ees (2w, >99\% ee; $\mathbf{2 x},>99 \%$ ee). Fluorene-derived alkene underwent desymmetrization and cyclization to generate product $\mathbf{2 z}$ efficiently as well.

Practicability of the Developed System. To test the generality of the developed method, alkene $\mathbf{3}$ with more flexible benzyl groups was examined under the similar conditions (Fig. 2a). Product 4 was formed in high yield with good enantioselectivity. When this method was applied to the desymmetrization and sulfenylation of 1a with sulfenylating reagents, no reaction occurred. This result was unexpected since the carbosulfenylation of alkenes has been realized by chiral selenophosphoramide catalysis ${ }^{66-68}$. Moreover, when olefinic diols were treated with sulfenylating reagent $\mathbf{6}$ in the presence of catalyst C7, thioproduct 7 was obtained in $67 \%$ with $92 \%$ ee and 9:1 $d r$ via desymmetrization (Fig. 2b). The result shows that the developed reaction system has great potential for electrophilic functionalization of alkenes with different electrophilic reagents, and thus will trigger more explorations using the similar conditions.

To further test the practical utility of the method, the reaction was scaled up with low catalyst loading. For example, desymmetrization of 1a $(1.0 \mathrm{~g})$ afforded product $2 \mathrm{a}(1.23 \mathrm{~g})$ in $99 \%$ yield with excellent enantioselectivity ( $>99 \%$ ee) using $2 \mathrm{~mol} \%$ C7 (Fig. 2c). This desymmetrization reaction could run at the room temperature, and was rapidly completed within $5 \mathrm{~min}$ using catalyst C7 to give product without much erosion of the selecticity. This result enhances the practicability of the method out of the lab. The recycle of the catalyst was also investigated. Alkene 1r was chosen as the substrate because of its easy separation from the catalyst (Fig. 2d). During the recycling, the product was obtained in high yield for each time, and its enantioselectivity remained unchanged. After being recycled five times, 92\% catalyst was still recovered.

The functional groups on substrates not only helped to enhance the selectivity of the reaction, but also offered us a great opportunity to pursue further transformations of products. Some synthetic applications of $\mathbf{2 a}$ are depicted in Fig. 3 and all the 
a
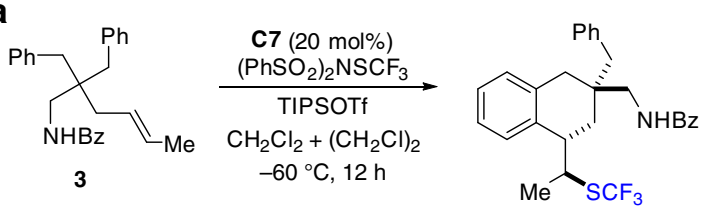

$4,80 \%, 74 \%$ ee, $d r=14: 1$

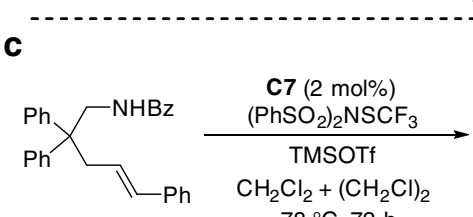

$1 \mathrm{a}, 1.0 \mathrm{~g}$

$-78^{\circ} \mathrm{C}, 72 \mathrm{~h}$

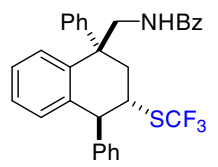

2a, $1.23 \mathrm{~g}$

$99 \%,>99 \%$ ee, $d r=50: 1$

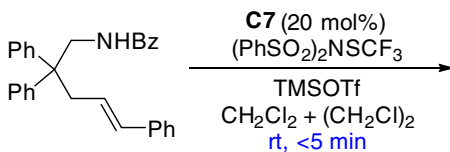

$1 a$

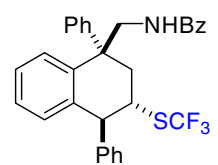

2a

$96 \%, 91 \%$ ee, $d r=5: 1$ \begin{tabular}{l} 
b \\
$\vdots$ \\
\hdashline \\
\hdashline \\
\hdashline
\end{tabular}

d
 $67 \%, 92 \%$ ee, $9: 1 d r$

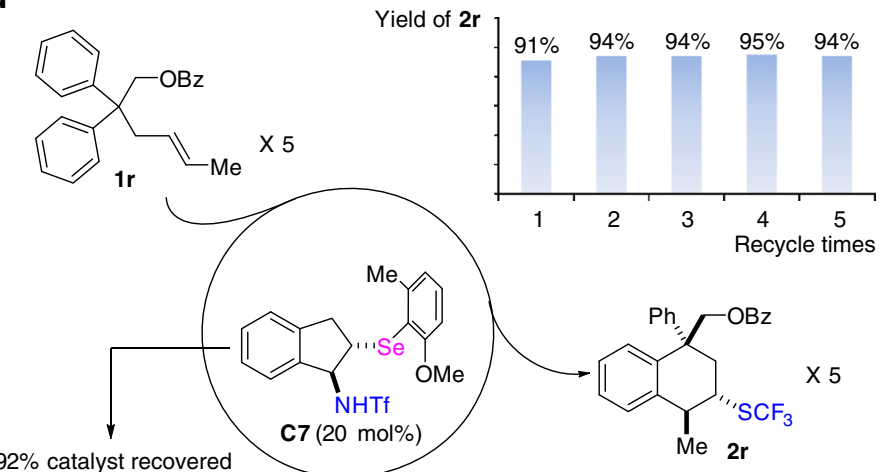

$94 \%$ average yield with $97 \%$ ee each

Fig. 2 Practicability of the developed system. a Transformation of substrate with flexible chain. b Desymmetrization and sulfenylation of diols. $\mathbf{c}$ Gram-scale reaction and reaction at room temperature. $\mathbf{d}$ Recycle of the catalyst

a<smiles>NC[C@]1(c2ccccc2)C[C@H](C(F)(F)F)C(c2ccccc2)c2ccccc21</smiles>

2ab, $96 \%$, 99\% ee<smiles>O=C(NCC1(c2ccccc2)CC=C(c2ccccc2)c2ccccc21)c1ccccc1</smiles>

2ae, $70 \%$, 99\% ee<smiles>O=C(NC[C@@]1(c2ccccc2)C[C@H](SC(F)(F)F)C(c2ccccc2)c2ccccc21)c1ccccc1</smiles>

2a

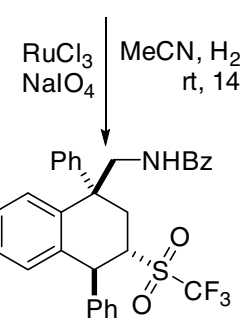

2ad, $99 \%$, 99\% ee<smiles>O=C([C@H]1C[C@@](CNC(=O)C(F)(F)F)(c2ccccc2)c2ccccc2C1c1ccccc1)[S+](=O)c1ccccc1</smiles>

2ac, $62 \%$, 99\% ee

b

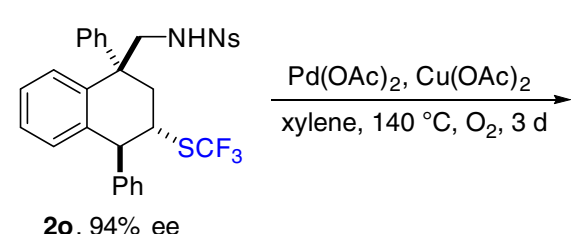

2o, $94 \%$ ee

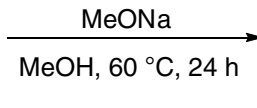<smiles>CS(=O)(=O)N1CC2(C[C@H](C(F)(F)F)[C@H](c3ccccc3)c3ccccc32)c2ccccc21</smiles>

2ag, 62\%, 94\% ee

Fig. 3 Further transformations of products. a Various transformations of $\mathbf{2 a}$. $\mathbf{b}$ Intramolecular Pd-catalyzed C-H amination of $\mathbf{2 0}$

derived compounds were isolated as single isomers. First, deprotection of benzoyl group on product 2 a gave a free amine $2 \mathrm{ab}$ in $96 \%$ yield. The $\mathrm{SCF}_{3}$ group could be oxidized to both $\mathrm{SOCF}_{3}$ and $\mathrm{SO}_{2} \mathrm{CF}_{3}$ groups by the appropriate oxidative systems. Compounds with $\mathrm{SO}_{2} \mathrm{CF}_{3}$ group could be further converted ${ }^{69-71}$. The generated 2ad easily underwent the elimination of triflic group to form alkene $2 \mathrm{ae}$ with $\mathrm{Me}_{3} \mathrm{SiOK}$. This provides a new route for the synthesis of valuable tetrahydronaphthalene derivatives, and shows a good potential of $\mathrm{SCF}_{3}$ group in synthetic utilities. Interestingly, $\mathbf{2} \mathbf{a f}$ was formed as a diastereoisomer from 2ad when $\mathrm{MeONa}$ was used as the base.
Furthermore, a spiroindoline derivative could be generated with 20 by an intramolecular $\mathrm{Pd}$-catalyzed $\mathrm{C}-\mathrm{H}$ amination. In the above-mentioned transformations, the erosion of enantioselectivity was not observed.

Computational Studies. During the reaction for the formation of 2a, a complex containing a chalcogenide-captured $\mathrm{CF}_{3} \mathrm{~S}$ cation was considered as the intermediate according to the work in which an active species was separated and could easily undergo the following step to afford the desired product for 


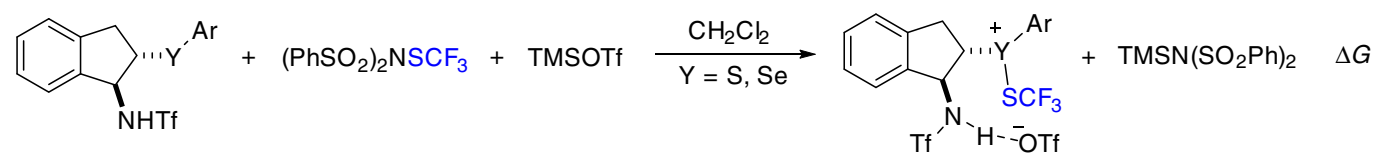

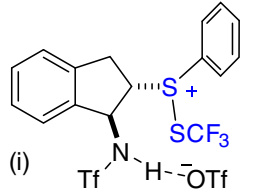

$\Delta G_{195 \mathrm{~K}}=+9.9 \mathrm{kcal} / \mathrm{mol}$

$\Delta H_{195 \mathrm{~K}}=+0.3 \mathrm{kcal} / \mathrm{mol}$

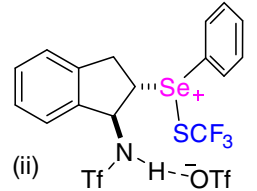

$\Delta G_{195 \mathrm{~K}}=+0.6 \mathrm{kcal} / \mathrm{mol}$

$\Delta H_{195 \mathrm{~K}}=-9.5 \mathrm{kcal} / \mathrm{mol}$
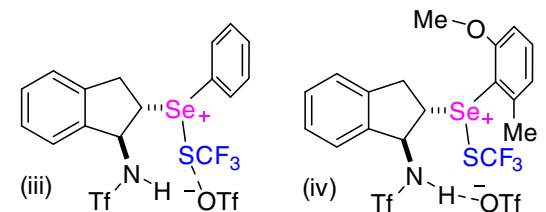

$\Delta G_{195 \mathrm{~K}}=+19.0 \mathrm{kcal} / \mathrm{mol} \quad \Delta G_{195 \mathrm{~K}}=+3.4 \mathrm{kcal} / \mathrm{mol}$

$\Delta H_{195 \mathrm{~K}}=+9.3 \mathrm{kcal} / \mathrm{mol}$

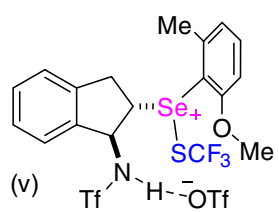

$\Delta G_{195 \mathrm{~K}}=-1.3 \mathrm{kcal} / \mathrm{mol}$

$\Delta H_{195 \mathrm{~K}}=-11.2 \mathrm{kcal} / \mathrm{mol}$

Fig. 4 Computational studies. Change of Gibbs free energy based on computational studies

enantioselective sulfenofunctionalization of alkenes ${ }^{61}$. The formation of this intermediate is the commencement of the reaction and can be affected by the used chalcogenide catalysts. On the basis of the experimental results in Table 1 and our previous studies $^{20}$, selenide catalysts are generally superior to the corresponding sulfide ones in promoting trifluoromethylthiolation, which reflects that selenides may activate $\mathrm{CF}_{3} \mathrm{~S}$-reagent easier to generate the ion pair intermediate than sulfides. To figure out the difference between sulfide and selenide catalysts, the impact of different catalysts on the formation of chalcogenide-captured $\mathrm{CF}_{3} \mathrm{~S}$ cation was investigated. Five models with different binding interactions were proposed and the change of Gibbs free energy reflecting the difference between selenide and sulfide catalysts was calculated (Fig. 4). The results of $\Delta G$ clearly showed the huge difference caused by different catalysts. With the aid of the additive acid, the free energy for the activation of $\mathrm{CF}_{3} \mathrm{~S}$ reagent by selenide is $+0.6 \mathrm{kcal} / \mathrm{mol}$ in an exothermic process, but $+9.9 \mathrm{kcal} /$ $\mathrm{mol}$ is needed to promote such step using sulfide catalyst (Fig. $4 \mathrm{i}$ and 4ii). When $\mathrm{TfO}^{-}$anion binds to the acidic proton of the catalyst, the energy for the formation of cationic complex is largely lowered (Fig. 4ii vs. 4iii). Furthermore, when the optimal catalyst C7 is utilized, the activation energy of the step is lowest when the methyl and methoxy groups are at the appropriate positions (Fig. 4v). These computational results match experimental ones, and indicate that high-energy barrier is required for sulfide catalysis in the initial activation step and selenide is better than sulfide in the activation of the electrophilic reagent.

Proposed Mechanism. On the basis of the above results and DFT calculations, a possible reaction pathway is proposed (Fig. 5a). First, selenide catalyst activates $\mathrm{CF}_{3} \mathrm{~S}$ reagent in the presence of Lewis acid to form intermediate int-I. Then, it reacts with substrate 1a to afford iranium ion int-III through transition state TSI, after which the phenyl ring on the chain attacks the iranium ion to form the final product $2 \mathrm{a}$. The reaction is spontaneous and exothermic according to calculating energies, which reasonably explains why the reaction is highly efficient under the optimal conditions. Considering the role of $\mathrm{TfO}^{-}$anion in the formation of int-I, an anion-binding interaction with a catalyst is proposed through the entire pathway. For substrate 1a with an $\mathrm{NHBz}$ group, an additional interaction between $\mathrm{TfO}^{-}$and the $\mathrm{NHBz}$ group is suggested to construct an anion bridge in the transformation based on DFT calculations. Interestingly, the proposed anion bridge can lower the energy of the intermediates. For example, when int-I directly binds to substrate $\mathbf{1 a}$ by hydrogen bonding, the formed intermediate has a higher energy of $2.3 \mathrm{kcal} /$ mol in comparison to int-II (for details, see Supplementary Fig. 179). Moreover, it is noteworthy that the anion-binding interaction with the catalyst may provide a good chance for acids to participate in the construction of the chiral environment of reaction. Especially, the effect may be more evident when the substrates without $\mathrm{H}$-bonding donor groups are utilized. Because of the anion-binding interaction with catalyst, the spatial hindrance of catalytic system is modified to further fix the absolute configuration of transition states. This can be the reason why products, e.g., $\mathbf{2 q}, \mathbf{2} \mathbf{r}$, and $\mathbf{2 s}$, without $\mathrm{H}$-bonding donor groups are generated in high enantioselectivities.

When calculating the reaction pathway of $\mathbf{1 a}$, it was found that the the highest energy appeared in different transition states for its four diastereomers. The highest energy is required for the attack of the phenyl ring toward the iranium ion to generate diastereomers $(1 R, 3 S, 4 S)-\mathbf{2 a}$ and $(1 R, 3 R, 4 R)-\mathbf{2 a}$. For the formation of the other two diastereomers, the highest energy barrier lies in the step of the iranium ion formation (Fig. 5a). On the basis of the Curtin-Hammett Principle ${ }^{72}$, the formation of TS-I and TS-II involves in the enantiodetermination of chiral centers. The energy for the formation of their possible transition states is compared (Fig. $5 \mathrm{~b})$. A relative $\Delta \Delta G(5.2 \mathrm{kcal} / \mathrm{mol})$ for TSI-SRR is obtained to predict the enantioselectivity of the major product. The predicted value is $99.9 \%$, which is close to the experimental result $(\mathbf{2 a},>99 \%$ ee). The energy discrepancy in transition states mainly comes from the perturbance of interaction and the distortion of catalyst and substrate (see the distortion-interaction analysis in Supplementary Table 4). Such two factors affect the energy of TS-II-RRR $(\Delta \Delta G=1.4 \mathrm{kcal} / \mathrm{mol})$ and TS-I-SSS $(\Delta \Delta G=3.3 \mathrm{kcal} / \mathrm{mol})$ as well, which result in different diastereomers of reaction $\left(d r_{\text {predicted }}=37: 1\right)$. Furthermore, DFT calculations for the formation of $2 \mathbf{q}$ without $\mathrm{H}$ bonding interaction between substrate and $\mathrm{TfO}^{-}$anion was also conducted based on the similar model. Similar results were obtained. By comparing the energy difference of the corresponding two transition states, TS-II'-RSS and TS-I'-SRR, the predicted enantioselectivity for the final product is $99.6 \%$ ee which is a little higher than the experimental result of $96 \%$ ee ( $\Delta \Delta \mathrm{G}=3.6 \mathrm{kcal} / \mathrm{mol}$, see Supplementary Fig. 176 for details).

\section{Discussion}

In summary, we have developed an efficient approach for enantioselective desymmetrization and carbotrifluoromethylthiolation of gem-diaryl-tethered alkenes and alkynes to form chiral trifluoromethylthiolated tetrahydronaphthalenes by a bifunctional selenide catalyst. The desired products were obtained with excellent enantio- and diastereoselectivities. They could be further converted under mild conditions, which provided new pathways for the synthesis of various valuable tetrahydronaphthalene derivatives. The developed reaction could be scaled up to gramscale and the catalytic system could also be used to the sulfenylation and desymmetrization of diols. These facts indicate that this method has great synthetic utility and practicality. Computational studies revealed the reason why selenide catalysis is more 


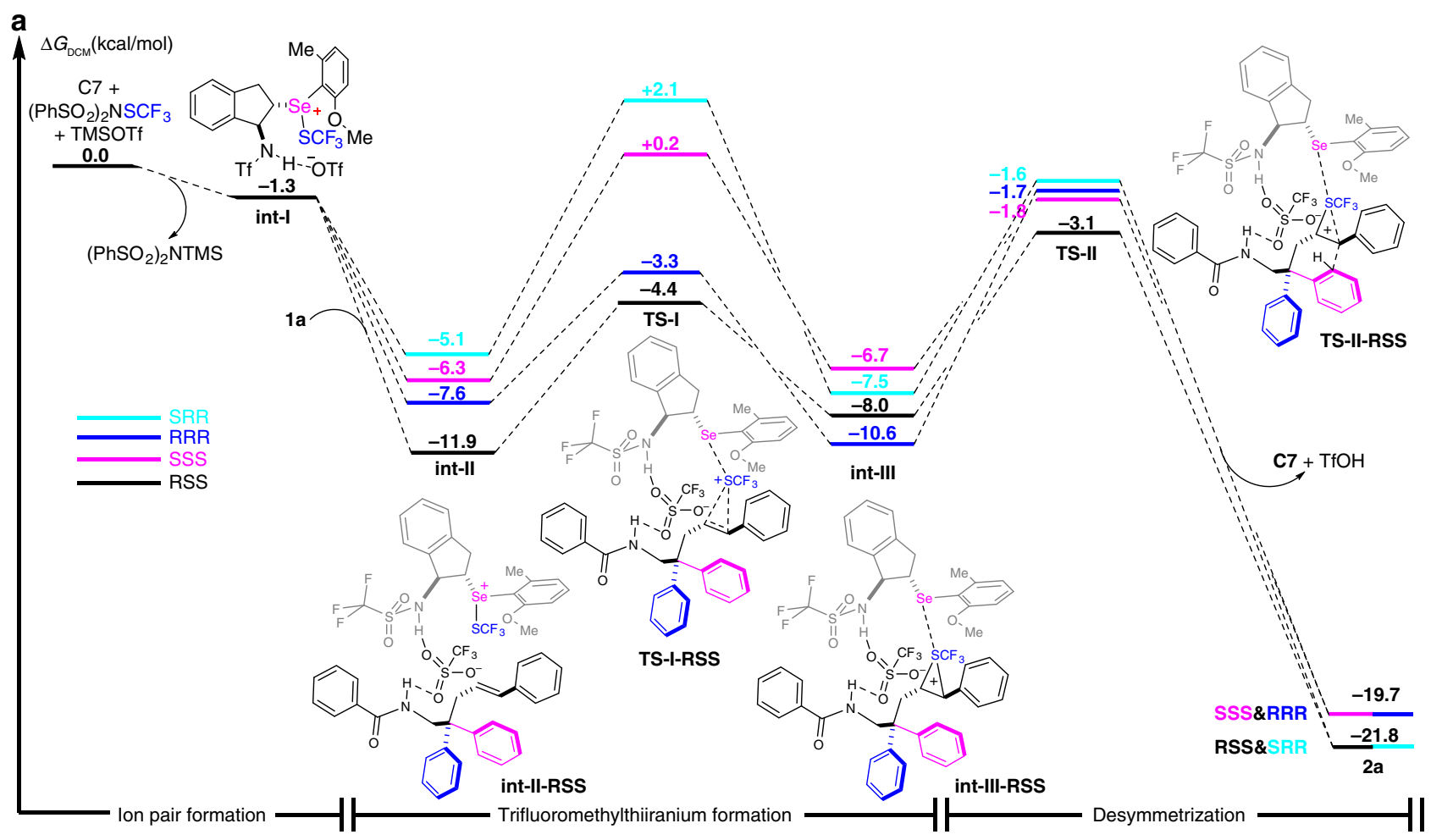

b

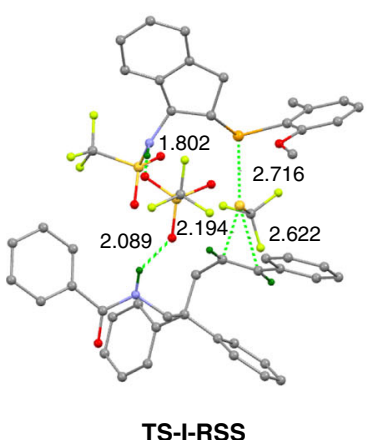

$\Delta \Delta G_{195 \mathrm{~K}}=-1.3 \mathrm{kcal} / \mathrm{mol}$

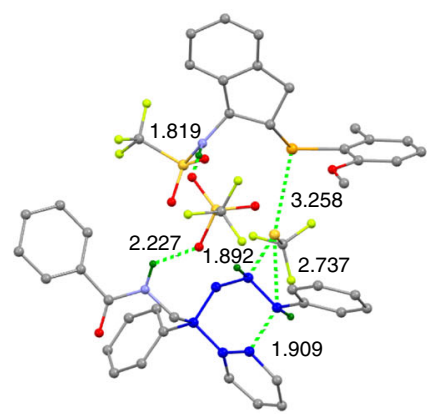

TS-II-RSS

$\Delta \Delta G_{195 \mathrm{~K}}=0.0 \mathrm{kcal} / \mathrm{mol}$

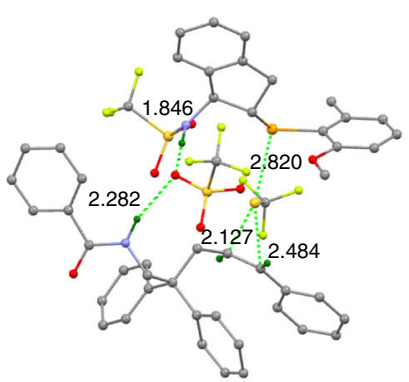

TS-I-RRR

$\Delta \Delta G_{195 \mathrm{~K}}=-0.2 \mathrm{kcal} / \mathrm{mol}$

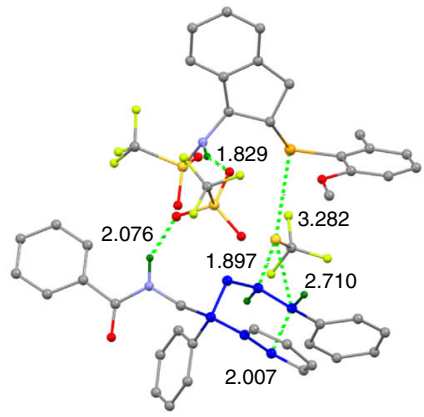

TS-II-RRR

$\Delta \Delta G_{195 \mathrm{~K}}=1.4 \mathrm{kcal} / \mathrm{mol}$

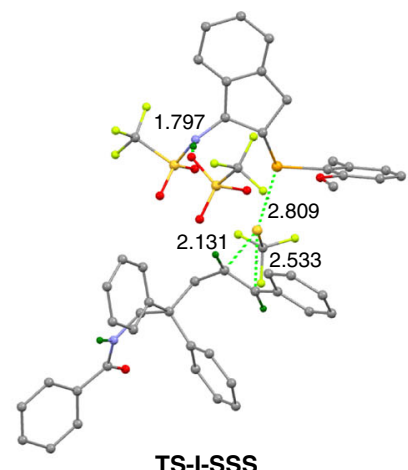

$\Delta \Delta G_{195 \mathrm{~K}}=3.3 \mathrm{kcal} / \mathrm{mol}$

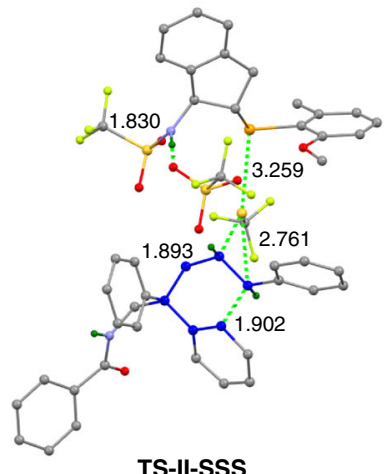

TS-II-SSS

$\Delta \Delta G_{195 \mathrm{~K}}=1.3 \mathrm{kcal} / \mathrm{mol}$

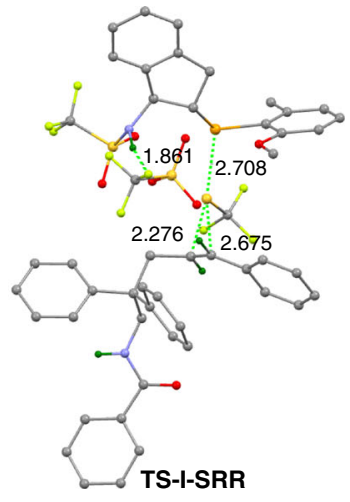

$\Delta \Delta G_{195 \mathrm{~K}}=5.2 \mathrm{kcal} / \mathrm{mol}$
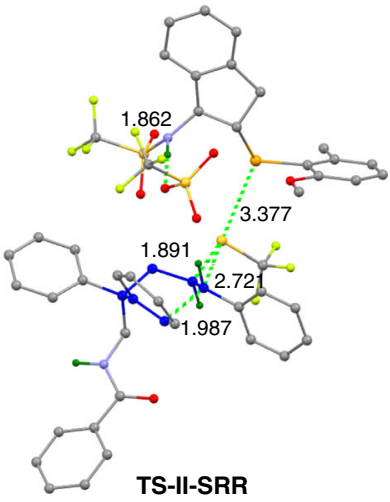

$\Delta \Delta G_{195 \mathrm{~K}}=1.5 \mathrm{kcal} / \mathrm{mol}$

Fig. 5 Proposed mechanism. a DFT calculations for reaction pathway at $195.15 \mathrm{~K}$. b Calculated transition states related to TS-I and TS-II

efficient than sulfide catalysis, and suggested an anion-binding interaction in the whole pathway. This work constitutes an additional strategy for the synthesis of chiral trifluoromethylthiolated molecules, highlights the efficiency of selenide catalysis, and is complementary to Lewis base catalysis.

\section{Methods}

Chiral Selenide-Catalyzed Desymmetrization. To a solution of olefin $(0.1$ $\mathrm{mmol}),\left(\mathrm{PhSO}_{2}\right)_{2} \mathrm{~N}-\mathrm{SCF}_{3}(59.8 \mathrm{mg}, 0.15 \mathrm{mmol})$, and catalyst C7 (9.3 $\left.\mathrm{mg}, 20 \mathrm{~mol} \%\right)$ in solvent $\left(\mathrm{CH}_{2} \mathrm{Cl}_{2} 2 \mathrm{ml},\left(\mathrm{CH}_{2} \mathrm{Cl}\right)_{2} 2 \mathrm{ml}\right)$ at $-78^{\circ} \mathrm{C}$ was added TMSOTf $(18.0 \mu \mathrm{\mu l}, 0.1$ mmol). The resultant mixture was stirred at $-78^{\circ} \mathrm{C}$ for $12 \mathrm{~h}$, and then quenched with $\mathrm{MeOH}(0.2 \mathrm{ml})$ and $\mathrm{Et}_{3} \mathrm{~N}(0.2 \mathrm{ml})$, and concentrated in vacuo. The residue 
was purified by flash silica gel column chromatography to yield the corresponding $\mathrm{CF}_{3} \mathrm{~S}$ product.

Chiral Selenide-Catalyzed Sulfenocyclization. To a solution of olefin $\mathbf{5}(17.8 \mathrm{mg}$, $0.1 \mathrm{mmol})$, saccharin- $\mathrm{S}(p-\mathrm{Tol})(36.6 \mathrm{mg}, 0.12 \mathrm{mmol})$ and catalyst $\mathbf{C} 7(9.3 \mathrm{mg}, 20$ mol\%) in solvent $\left(\mathrm{CH}_{2} \mathrm{Cl}_{2} 4 \mathrm{ml}\right)$ at $-78^{\circ} \mathrm{C}$ was added TMSOTf (18.0 $\left.\mu \mathrm{l}, 0.1 \mathrm{mmol}\right)$. The resultant mixture was stirred at $-78^{\circ} \mathrm{C}$ for $12 \mathrm{~h}$, and then quenched by saturated $\mathrm{NaHCO}_{3}(1 \mathrm{ml})$ and then extracted with dichloromethane $(8 \mathrm{ml} \times 4)$. The combined organic phases were concentrated in vacuo. The residue was purified by flash silica gel column chromatography to yield the corresponding thioproduct 7 (67\%, 92\% ee, 9:1 dr)

For nuclear magnetic resonance and high-performance liquid chromatography spectra, see Supplementary Figs 7-169.

Data Availability. The X-ray crystallographic coordinates for structures reported in this article have been deposited at the Cambridge Crystallographic Data Centre (CCDC), under deposition numbers CCDC 1523336, 1577179, 1532614, 1533403, and 1540104. The data can be obtained free of charge from The Cambridge Crystallographic Data Centre via http://www.ccdc.cam.ac.uk/data_request/cif. Any further relevant data are available from the authors upon reasonable request.

Received: 11 July 2017 Accepted: 9 January 2018

Published online: 06 February 2018

\section{References}

1. Jeschke, P. The unique role of fluorine in the design of active ingredients for modern crop protection. Chembiochem 5, 570-589 (2004).

2. Liang, T., Neumann, C. N. \& Ritter, T. Introduction of fluorine and fluorinecontaining functional groups. Angew. Chem. Int. Ed. 52, 8214-8264 (2013).

3. Yang, X., Wu, T., Phipps, R. J. \& Toste, F. D. Advances in catalytic enantioselective fluorination, mono-,di-, and trifluoromethylation, and trifluoromethylthiolation reactions. Chem. Rev. 115, 826-870 (2015).

4. Manteau, B. et al. New trends in the chemistry of $\alpha$-fluorinated ethers, thioethers, amines and phosphines. J. Fluor. Chem. 131, 140-158 (2010).

5. Toulgoat, F., Alazet, S. \& Billard, T. Direct trifluoromethylthiolation reactions: the "renaissance" of an old concept. Eur. J. Org. Chem. 2014, 2415-2428 (2014).

6. Xu, X.-H., Matsuzaki, K. \& Shibata, N. Synthetic methods for compounds having $\mathrm{CF}_{3}-\mathrm{S}$ units on carbon by trifluoromethylation, trifluoromethylthiolation, triflylation, and related reactions. Chem. Rev. 115, 731-764 (2015).

7. Ferry, A., Billard, T., Langlois, B. R. \& Bacqué, E. Trifluoromethanesulfanylamides as easy-to-handle equivalents of the trifluoromethanesulfanyl cation $\left(\mathrm{CF}_{3} \mathrm{~S}^{+}\right)$: reaction with alkenes and alkynes. Angew. Chem. Int. Ed. 48, 8551-8555 (2009).

8. Chen, C., Chu, L. \& Qing, F.-L. Metal-free oxidative trifluoromethylthiolation of terminal alkynes with $\mathrm{CF}_{3} \mathrm{SiMe}_{3}$ and elemental sulfur. J. Am. Chem. Soc. 134, 12454-12457 (2012).

9. Yang, Y.-D. et al. Trifluoromethanesulfonyl hypervalent iodonium ylide for copper-catalyzed trifluoromethylthiolation of enamines, indoles, and $\beta$-keto esters. J. Am. Chem. Soc. 135, 8782-8785 (2013).

10. Danoun, G., Bayarmagnai, B., Gruenberg, M. F. \& Goossen, L. J. Sandmeyer trifluoromethylthiolation of arenediazonium salts with sodium thiocyanate and Ruppert-Prakash reagent. Chem. Sci. 5, 1312-1316 (2014).

11. Guo, S., Zhang, X. \& Tang, P. Silver-mediated oxidative aliphatic C-H trifluoromethylthiolation. Angew. Chem. Int. Ed. 54, 4065-4069 (2015).

12. Mukherjee, S., Maji, B., Tlahuext-Aca, A. \& Glorius, F. Visible-light-promoted activation of unactivated $\mathrm{C}\left(s p^{3}\right)-\mathrm{H}$ bonds and their selective trifluoromethylthiolation. J. Am. Chem. Soc. 138, 16200-16203 (2016).

13. Bootwicha, T. et al. $N$-Trifluoromethylthiophthalimide: a stable electrophilic $\mathrm{SCF}_{3}$-reagent and its application in the catalytic asymmetric trifluoromethylsulfenylation. Angew. Chem. Int. Ed. 52, 12856-12859 (2013).

14. Wang, X., Yang, T., Cheng, X. \& Shen, Q. Enantioselective electrophilic trifluoromethylthiolation of $\beta$-ketoesters: a case of reactivity and selectivity bias for organocatalysis. Angew. Chem. Int. Ed. 52, 12860-12864 (2013).

15. Zhu, X.-L. et al. In situ generation of electrophilic trifluoromethylthio reagents for enantioselective trifluoromethylthiolation of oxindoles. Org. Lett. 16, 2192-2195 (2014).

16. Deng, Q.-H. et al. Copper-boxmi complexes as highly enantioselective catalysts for electrophilic trifluoromethylthiolations. Chem. Eur. J. 20, 93-97 (2014).

17. Zhao, B.-L. \& Du, D.-M. Enantioselective squaramide-catalyzed trifluoromethylthiolation-sulfur-Michael/Aldol cascade reaction: one-pot synthesis of $\mathrm{CF}_{3} \mathrm{~S}$-containing spiro cyclopentanone-thiochromanes. Org. Lett. 19, 1036-1039 (2017).
18. Zhang, Z. et al. Catalytic asymmetric trifluoromethylthiolation via enantioselective $[2,3]$-sigmatropic rearrangement of sulfonium ylides. Nat. Chem. 9, 970-976 (2017).

19. Liu, X. et al. Enantioselective trifluoromethylthiolating lactonization catalyzed by an indane-based chiral sulfide. Angew. Chem. Int. Ed. 55, 5846-5850 (2016).

20. Luo, J., Liu, Y. \& Zhao, X. Chiral selenide-catalyzed enantioselective construction of saturated trifluoromethylthiolated azaheterocycles. Org. Lett. 19, 3434-3437 (2017)

21. Enríquez-Garcíawand, Á. \& Kündig, E. P. Desymmetrisation of meso-diols mediated by non-enzymatic acyl transfer catalysts. Chem. Soc. Rev. 41, 7803-7831 (2012)

22. Petersen, K. S. Nonenzymatic enantioselective synthesis of all-carbon quaternary centers through desymmetrization. Tetrahedron Lett. 56, 6523-6535 (2015).

23. Zeng, X.-P. et al. Catalytic enantioselective desymmetrization reactions to allcarbon quaternary stereocenters. Chem. Rev. 116, 7330-7396 (2016).

24. Lee, J. Y., You, Y. S. \& Kang, S. H. Asymmetric synthesis of all-carbon quaternary stereocenters via desymmetrization of 2,2-disubstituted 1,3propanediols. J. Am. Chem. Soc. 133, 1772 (2011).

25. Aikawa, K., Okamoto, T. \& Mikami, K. Copper(I)-catalyzed asymmetric desymmetrization: Synthesis of five-membered-ring compounds containing all-carbon quaternary stereocenters. J. Am. Chem. Soc. 134, 10329-10332 (2012).

26. Saget, T. \& Cramer, N. Enantioselective C-H arylation strategy for functionalized dibenzazepinones with quaternary stereocenters. Angew. Chem. Int. Ed. 52, 7865-7868 (2013).

27. Zhao, Y., Jiang, X. \& Yeung, Y.-Y. Catalytic, enantioselective, and highly chemoselective bromocyclization of olefinic dicarbonyl compounds. Angew. Chem. Int. Ed. 52, 8597-8601 (2013).

28. Cheng, X.-F. et al. Pd(II)-catalyzed enantioselective C-H activation/C-O bond formation: synthesis of chiral benzofuranones. J. Am. Chem. Soc. 135, 1236-1239 (2013).

29. Meng, S.-S. et al. Chiral phosphoric acid catalyzed highly enantioselective desymmetrization of 2-substituted and 2,2-disubstituted 1,3-diols via oxidative cleavage of benzylidene acetals. J. Am. Chem. Soc. 136, 12249-12252 (2014).

30. Partridge, B. M., González, J. S. \& Lam, H. W. Iridium-catalyzed arylative cyclization of alkynones by 1,4-iridium migration. Angew. Chem. Int. Ed. 53, 6523-6527 (2014)

31. Yang, W., Liu, Y., Zhang, S. \& Cai, Q. Copper-catalyzed intramolecular desymmetric aryl C-O coupling for the enantioselective construction of chiral dihydrobenzofurans and dihydrobenzopyrans. Angew. Chem. Int. Ed. 54, 8805-8808 (2015).

32. Wang, Z. et al. Catalytic enantioselective intermolecular desymmetrization of azetidines. J. Am. Chem. Soc. 137, 5895-5898 (2015).

33. Wilking, M., Mück-Lichtenfeld, C., Daniliuc, C. G. \& Hennecke, U. Enantioselective, desymmetrizing bromolactonization of alkynes. J. Am. Chem. Soc. 135, 8133-8136 (2013).

34. Wilking, M., Daniliuc, C. G. \& Hennecke, U. Monomeric cinchona alkaloidbased catalysts for highly enantioselective bromolactonisation of alkynes. Chem. Eur. J. 22, 18601-18607 (2016).

35. Arai, M. A., Kuraishi, M., Arai, T. \& Sasai, H. A new asymmetric Wacker-type cyclization and tandem cyclization promoted by $\mathrm{Pd}(\mathrm{II})$-spiro bis(isoxazoline) catalyst. J. Am. Chem. Soc. 123, 2907-2908 (2001)

36. Seiser, T. \& Cramer, N. Enantioselective C-C bond activation of allenyl cyclobutanes: access to cyclohexenones with quaternary stereogenic centers. Angew. Chem. Int. Ed. 47, 9294-9297 (2008).

37. Tay, D. W., Leung, G. Y. C. \& Yeung, Y.-Y. Desymmetrization of diolefinic diols by enantioselective aminothiocarbamate-catalyzed bromoetherification: synthesis of chiral spirocycles. Angew. Chem. Int. Ed. 53, 5161-5164 (2014).

38. Ke, Z., Tan, C., Chen, K. \& Yeung, Y.-Y. Catalytic asymmetric bromoetherification and desymmetrization of olefinic 1,3-diols with $\mathrm{C}_{2}$ symmetric sulfides. J. Am. Chem. Soc. 136, 5627-5630 (2014).

39. Zi, W. \& Toste, F. D. Gold(I)-catalyzed enantioselective desymmetrization of 1,3-diols through intramolecular hydroalkoxylation of allenes. Angew. Chem. Int. Ed. 54, 14447-14451 (2015).

40. Gu, Q. \& You, S.-L. Desymmetrization of cyclohexadienonesviacinchonine derived thiourea-catalyzed enantioselective aza-Michael reaction and total synthesis of (-)-Mesembrine. Chem. Sci. 2, 1519-1522 (2011).

41. Mourad, A. K., Leutzow, J. \& Czekelius, C. Anion-induced enantioselective cyclization of diynamides to pyrrolidines catalyzed by cationic gold complexes. Angew. Chem. Int. Ed. 51, 11149-11152 (2012).

42. Babij, N. R. \& Wolfe, J. P. Desymmetrization of meso-2,5-diallylpyrrolidinyl oreas through asymmetric palladium-catalyzed carboamination: stereocontrolled synthesis of bicyclic ureas. Angew. Chem. Int. Ed. 52, 9247-9250 (2013)

43. Manna, K., Eedugurala, N. \& Sadow, A. D. Zirconium-catalyzed desymmetrization of aminodialkenes and aminodialkynes through enantioselective hydroamination. J. Am. Chem. Soc. 137, 425-435 (2015). 
44. Ward, R. S. Lignans, neolignans and related compounds. Nat. Prod. Rep. 16, 75-96 (1999).

45. Razzakov, N. A., Vakhabov, A. \& Aripova, S. F. Quaternary derivatives of $\alpha$ and $\beta$-scopodonnines and their pharmacologic activity. Chem. Nat. Compd. 39, 215-217 (2003).

46. Gordaliza, M. et al. Podophyllotoxin: distribution, sources, applications and new cytotoxic derivatives. Toxicon 44, 441-459 (2004).

47. Miller, B. \& Shi, X. Cyclization and rearrangement processes resulting from bromination of 3-benzylcycloalkenes. J. Org. Chem. 58, 2907-2909 (1993).

48. Haro, T. \& Nevado, C. Flexible gold-catalyzed regioselective oxidative difunctionalization of unactivated alkenes. Angew. Chem. Int. Ed. 50, 906-910 (2011).

49. Cacciuttolo, B. et al. Access to polycyclic derivatives by triflate-catalyzed intramolecular hydroarylation. Eur. J. Org. Chem. 2014, 7458-7468 (2014).

50. Zeng, W. \& Chemler, S. R. Copper(II)-catalyzed enantioselective intramolecular carboamination of alkenes. J. Am. Chem. Soc. 129, 12948-12949 (2007).

51. Miao, L. et al. Diastereo- and enantioselective copper-catalyzed intramolecular carboamination of alkenes for the synthesis of hexahydro-1H-benz[f]indoles. Org. Lett. 12, 4739-4741 (2010).

52. Miller, Y., Miao, L., Hosseini, A. S. \& Chemler, S. R. Copper-catalyzed intramolecular alkene carboetherification: synthesis of fused-ring and bridgedring tetrahydrofurans. J. Am. Chem. Soc. 134, 12149-12156 (2012).

53. Bovino, M. T. et al. Enantioselective copper-catalyzed carboetherification of unactivated alkenes. Angew. Chem. Int. Ed. 53, 6383-6387 (2014).

54. Vedejs, E., Denmark, S. E. (eds). Lewis Base Catalysis in Organic Synthesis. (Wiley-VCH, Weinheim, 2016).

55. Braga, A. L., Lüdtke, D. S., Vargas, F. \& Braga, R. C. Catalytic applications of chiral organoselenium compounds in asymmetric synthesis. Synlett 37, 1453-1466 (2006).

56. Braga, A. L., Lüdtke, D. S. \& Vargas, F. Enantioselective synthesis mediated by catalytic chiral organoselenium compounds. Curr. Org. Chem. 10, 1921-1938 (2006).

57. Denmark, S. E. \& Collins, W. R. Lewis base activation of lewis acids: development of a Lewis base catalyzed selenolactonization. Org. Lett. 9, 3801-3804 (2007).

58. Denmark, S. E. \& Beutner, G. L. Lewis base catalysis in organic synthesis. Angew. Chem. Int. Ed. 47, 1560-1638 (2008).

59. Denmark, S. E., Kalyani, D. \& Collins, W. R. Preparative and mechanistic studies toward the rational development of catalytic, enantioselective selenoetherification reactions. J. Am. Chem. Soc. 132, 15752-15765 (2010).

60. Denmark, S. E. \& Chi, H. M. Lewis base catalyzed, enantioselective, intramolecular sulfenoamination of olefins. J. Am. Chem. Soc. 136, 8915-8918 (2014).

61. Denmark, S. E., Hartmann, E., Kornfilt, D. J. P. \& Wang, H. Mechanistic, crystallographic, and computational studies on the catalytic, enantioselective sulfenofunctionalization of alkenes. Nat. Chem. 6, 1056-1064 (2014).

62. Chen, F., Tan, C. K. \& Yeung, Y.-Y. $C_{2}$-symmetric cyclic selenium-catalyzed enantioselective bromoaminocyclization. J. Am. Chem. Soc. 135, 1232-1235 (2013).

63. Luo, J., Zhu, Z., Liu, Y. \& Zhao, X. Diaryl selenide catalyzed vicinal trifluoromethylthioamination of alkenes. Org. Lett. 17, 3620-3623 (2015).

64. Wu, J.-J., Xu, J. \& Zhao, X. Selenide-catalyzed stereoselective construction of tetrasubstituted trifluoromethylthiolated alkenes with alkynes. Chem. Eur. J. 22, 15265-15269 (2016).

65. Luo, J., Liu, X. \& Zhao, X. Development of chalcogenide catalysts towards trifluoromethylthiolation. Synlett 28, 397-401 (2017).

66. Denmark, S. E. \& Jaunet, A. Catalytic, enantioselective, intramolecular carbosulfenylation of olefins. J. Am. Chem. Soc. 135, 6419-6422 (2013).

67. Denmark, S. E. \& Chi, H. M. Catalytic, enantioselective, intramolecular carbosulfenylation of olefins. Mechanistic aspects: a remarkable case of negative catalysis. J. Am. Chem. Soc. 136, 3655-3663 (2014).
68. Denmark, S. E. \& Jaunet, A. Catalytic, enantioselective, intramolecular carbosulfenylation of olefins. Preparative and stereochemical aspects. J. Org. Chem. 79, 140-171 (2014).

69. Hendrickson, J. B., Gigs, A. \& Wareing, J. Triflones $\left(\mathrm{CF}_{3} \mathrm{SO}_{2} \mathrm{C}\right)$. A survey of reactivity and synthetic utility. J. Am. Chem. Soc. 96, 2275-2276 (1974).

70. Hendrickson, J. B., Bair, K. W. \& Keehn, P. M. Conversion of triflones to ketones. J. Org. Chem. 42, 2935-2936 (1977).

71. Hellmann, G. et al. Chiral fluorinateda-sulfonyl carbanions: Enantioselective synthesis and electrophilic capture, racemization dynamics, and structure. Chem. Eur. J. 19, 3869-3897 (2013).

72. Seeman, J. I. The Curtin-Hammett priciple and the Winstein-Holness equation: new definition and recent extensions to classical concepts. J. Chem. Ed. 63, 42-48 (1986).

\section{Acknowledgements}

We thank Sun Yat-Sen University, the "One Thousand Youth Talents" Program of China and the Natural Science Foundation of Guangdong Province (Grant No.

2014A030312018) for financial support. We are grateful to our teammate, Dr. Jinji Wu, for single crystal structure analysis. We thank National Supercomputing Center in

Shenzhen for providing computer service for our computational studies. We also thank Professor Vy Dong at UCI for the great suggestions about the manuscript.

\section{Author contributions}

J.L. started and performed the experiments and prepared Supplementary Information. Q. C. performed additional experiments with respect to substrate scope. X.C. performed the computational studies and revised the paper. X.Z. conceived and directed the project and wrote the manuscript.

\section{Additional information}

Supplementary Information accompanies this paper at https://doi.org/10.1038/s41467018-02955-0.

Competing interests: The authors declare no competing financial interests.

Reprints and permission information is available online at http://npg.nature.com/ reprintsandpermissions/

Publisher's note: Springer Nature remains neutral with regard to jurisdictional claims in published maps and institutional affiliations.

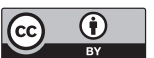

Open Access This article is licensed under a Creative Commons Attribution 4.0 International License, which permits use, sharing, adaptation, distribution and reproduction in any medium or format, as long as you give appropriate credit to the original author(s) and the source, provide a link to the Creative Commons license, and indicate if changes were made. The images or other third party material in this article are included in the article's Creative Commons license, unless indicated otherwise in a credit line to the material. If material is not included in the article's Creative Commons license and your intended use is not permitted by statutory regulation or exceeds the permitted use, you will need to obtain permission directly from the copyright holder. To view a copy of this license, visit http://creativecommons.org/ licenses/by/4.0/.

(c) The Author(s) 2018 